\title{
INCIDÊNCIA E REPERCUSSÃO DO CÂNCER DE COLO UTERINO EM MULHERES NEGRAS NO MUNICÍPIO DE QUEIMADOS - RJ
}

\author{
INCIDENCE AND REPERCUSSION OF CERVICAL CANCER IN BLACK WOMEN IN THE \\ MUNICIPALITY OF QUEIMADOS - RJ
}

\section{INCIDENCIA Y REPERCUSIÓN DEL CÁNCER CERVICAL EN MUJERES NEGRAS EN EL MUNICIPIO DE QUEIMADOS - RJ}

\begin{abstract}
Leandro Mendes Martins ${ }^{1}$, Adriana de Aquino Oliveira ${ }^{2}$, Maria Clara Veiga da Rocha ${ }^{3}$, Amanda de Almeida Floriano ${ }^{4}$, Daniel Virginio ${ }^{5}$, Bruna Porath Azevedo Fassarella ${ }^{6}$, Wanderson Alves Ribeiro ${ }^{7}$, Vanessa Vicente Alves Coutinho 8
\end{abstract}

Submetido em: 20/04/2021

e24227

Aprovado em: 30/04/2021

\section{RESUMO}

O debate sobre a saúde da mulher negra não tem sido um campo relevante ou uma área de conhecimento que venha despertando tanto interesse nas Ciências da Saúde. A produção de conhecimento científico nessa área é inexpressiva e essa temática não participa do currículo dos diferentes cursos de graduação e pós-graduação em saúde, com raríssimas exceções. Logicamente o tema em questão acaba sendo ignorado por grande parte de pesquisadores, estudantes e profissionais de saúde no Brasil. O presente estudo versou descrever a experiência frente ao índice de câncer do colo uterino em mulheres negras de 25 a 64 anos. Constatou-se que os entraves institucionais oriundos das questões de gestão tornam dificultoso a obtenção do cuidado, associados a esse fator, as características locais de cidade dormitório e o déficit de conhecimento acerca da periodicidade para realização de exames preventivos diferem entre mulheres negras e brancas. A adoção de medidas, como expansão de cobertura territorial em Saúde da Família, construção de vínculo entre profissional e usuários, implementação da Área Técnica de Atenção Integral a Saúde da População Negra e o uso da Educação em Saúde como ferramenta primordial de Promoção a Saúde foram alternativas encontradas para atenuar as disparidades existentes.

PALAVRAS-CHAVE: Saúde da Mulher Negra. Racismo. Racismo Institucional. Políticas Públicas e Educação em Saúde.

\footnotetext{
1 Especialista em Gestão de Atenção a Rede de Saúde - ENSP Sérgio Arouca - Fiocruz; Especialista em Saúde da Família - Escola de Enfermagem Luisa de Marilac - São Camilo, Enfermeiro da Equipe de Consultório na Rua pela Prefeitura Municipal de Nova Iguaçu - PMCNI e Enfermeiro da Clínica da Familia Irlan de Souza Macedo pela Prefeitura Municipal de Queimados

2 Enfermeira. Grupo Santa Celina Integradora de Saúde

3 Enfermeira. Bacharel em Enfermagem pelo Associação Brasileira de Ensino Universitário (UNIABEU)

${ }^{4}$ Enfermeira. Graduada pela Universidade Iguaçu.

${ }^{5}$ Médico de Família na Prefeitura Municipal de Queimados.

6 Enfermeira. Mestre em Urgência e Emergência pelo Programa de Ciências Aplicadas a Saúde da Universidade de Vassouras (FUSVE). Docentes dos cursos de graduação e pós-graduação graduação em enfermagem da Universidade Iguaçu (UNIG). Acadêmica em Medicina pela Universidade Iguaçu (UNIG) 7 Enfermeiro. Mestre e Doutorando pela Programa Acadêmico em Ciências do Cuidado em Saúde (PACCS) da Universidade federal Fluminense (UFF); Docentes dos cursos de graduação e pós-graduação graduação em enfermagem da Universidade Iguaçu (UNIG). Acadêmico em Medicina pela Universidade Iguaçu (UNIG). 8 Acadêmica em Psicologia pela Universidade do Grande Rio Professor José de Souza Herdy (UNIGRANRIO).
} 
RECIMA21 - REVISTA CIENTÍFICA MULTIDISCIPLINAR ISSN 2675-6218

ABSTRACT

The debate on the health of black women has not been a relevant field or an area of knowledge that has aroused so much interest in Health Sciences. The production of scientific knowledge in this area is inexpressive and this theme does not participate in the curriculum of different undergraduate courses and postgraduate studies in health, with very rare exceptions. Logically, the topic in question ends up being ignored by a large part of researchers, students and health professionals in Brazil. The present study aimed to describe the experience regarding the cervical cancer index in black women between 25 and 64 years old. It was found that the institutional barriers arising from management issues make it difficult to obtain care, associated with this factor, the local characteristics of a dormitory city and the lack of knowledge about the frequency of preventive exams differ between black and white women. The adoption of measures, such as the expansion of territorial coverage in Family Health, the construction of bonds between professionals and users, and the implementation of the Technical Area of Comprehensive Health Care for the Black Population and the use of Health Education as a primary tool for Health Promotion were alternatives found to mitigate the existing disparities.

KEYWORDS: Black Woman Health. Racism. Institutional Racism. Public Policies and Health Education.

\section{RESUMEN}

El debate sobre la salud de la mujer negra no ha sido un campo relevante o un área de conocimiento que haya despertado tanto interés en las Ciencias de la Salud. La producción de conocimiento científico en esta área es inexpresiva y esta temática no participa en el currículo. de diferentes carreras y posgrados en salud, con muy raras excepciones. Lógicamente, el tema en cuestión acaba siendo ignorado por gran parte de los investigadores, estudiantes y profesionales de la salud en Brasil. El presente estudio tuvo como objetivo describir la experiencia con respecto al índice de cáncer de cuello uterino en mujeres negras entre 25 y 64 años. Se encontró que las barreras institucionales que surgen por cuestiones de gestión dificultan la obtención de atención, asociado a este factor, las características locales de una ciudad dormitorio y el desconocimiento sobre la frecuencia de los exámenes preventivos difieren entre mujeres negras y blancas. La adopción de medidas, como la ampliación de la cobertura territorial en Salud de la Familia, la construcción de vínculos entre profesionales y usuarios, la implementación del Área Técnica de Atención Integral a la Salud de la Población Negra y el uso de la Educación para la Salud como herramienta de Promoción de la Salud fueron alternativas encontradas para mitigar las disparidades existentes.

DESCRIPTORES: Salud de la Mujer Negra. Racismo. Racismo institucional. Políticas públicas y educación para la salud.

\section{INTRODUÇÃO}

A inquietude por essa temática emergiu após rotina laborativa em uma unidade de estratégia saúde da família, situada no Município de Queimados, integrante da Região Metropolitana I, no Estado do Rio de Janeiro, onde percebeu-se que as usuárias cadastradas, conforme faixa etária preconizada para realização exame Papanicolau, que procuravam o serviço de Saúde da Mulher possuía um quantitativo de mulheres brancas proporcionalmente maior do que as negras. 


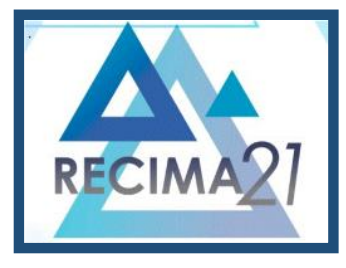

\title{
RECIMA21 - REVISTA CIENTÍFICA MULTIDISCIPLINAR ISSN 2675-6218
}

\author{
INCIDÊNCIA E REPERCUSSÃO DO CÂNCER DE COLO UTERINO EM MULHERES NEGRAS \\ NO MUNICÍPIO DE QUEIMADOS - RJ \\ Leandro Mendes Martins, Adriana de Aquino Oliveira, Maria Clara Veiga da Rocha, Amanda de Almeida Floriano, \\ Daniel Virginio, Bruna Porath Azevedo Fassarella, Wanderson Alves Ribeiro, Vanessa Vicente Alves Coutinho
}

Dentro dessa perspectiva a opção pela temática dá-se pela correlação da dualidade educação - saúde, fator primordial para aquisição de conhecimentos e empoderamento da mulher, sobretudo as mulheres negras, no binômio saúde-qualidade de vida, que pode ser diretamente afetado pelas desigualdades sociais existentes em nosso país.

$\mathrm{Na}$ sociedade brasileira, brancos, negros - pretos e pardos - e indígenas ocupam espaços sociais distintos, que se refletem nos indicadores sociais: negros e indígenas possuem os piores indicadores de escolaridade, estão inseridos nos piores postos de trabalho e têm um acesso menor a bens e serviços sociais. Estas desigualdades levam à miséria material, isolamento espacial e social, e restrições à participação política. Este processo, denominado racismo, enraizou-se na cultura, no tecido social e nos comportamentos da sociedade brasileira (MUNANGA, 2006).

Corroborando com a temática, Werneck (2005) afirma que o racismo é um fenômeno ideológico, um importante fator de violação de direitos e de produção de iniquidades, especialmente no campo da saúde. Ainda segundo ele o racismo tem uma relação com as condições em que a pessoa nasce, com sua trajetória familiar e individual, condições de vida e moradia, condições de trabalho, emprego, renda e de acesso à informação e aos bens e serviços. O racismo também é visível na qualidade do cuidado e assistência prestada, nos perfis e estimativa de mortalidade infantil, nos sofrimentos evitáveis ou mortes precoces, nas taxas de mortalidade da população adulta e nos perfis, indicadores e coeficientes de mortalidade materna.

Para Cordeiro e Ferreira (2009) a discriminação racial e de gênero, além do racismo institucional repercutem negativamente no acesso e na permanência das mulheres negras nos serviços de saúde e não estão inseridas apenas nas práticas individuais, mas fazem parte das normas e rotinas institucionais. Estudos têm demonstrado que as mulheres negras estão expostas a tratamentos inadequados e ações insuficientes de cuidado e prevenção em saúde, principalmente no âmbito da saúde sexual e reprodutiva (TULLER, 2008).

Nesse contexto, o INCA (2016) diz que o câncer de colo uterino (também chamado de cervical), que costuma acometer mulheres de classes mais abastardas da sociedade, é causado pela infecção persistente por alguns tipos (chamados oncogênicos) do Papilomavírus Humano HPV, podendo ser detectada facilmente através do exame preventivo, também conhecido como Papanicolau, sendo curável quase a totalidade dos casos.

Apesar do bom prognóstico, esse é o terceiro tumor mais comum na população feminina e suas taxas de mortalidade são elevadas, constituindo-se em um grave problema de Saúde Pública. A série histórica divulgada pelo Ministério da Saúde contempla dados que vão de 1979 a 2005. Nesse período as taxas de mortalidade ajustadas por idade passaram de 4,97 para 5,29 por 100.000 mulheres, o que representa um incremento de 6,4\% em 26 anos (BRASIL, 2008).

Para Chor (2015) as piores condições de vida e o menor acesso a serviços de saúde de qualidade fazem com que a incidência de determinados tipos de neoplasias sejam maiores - 


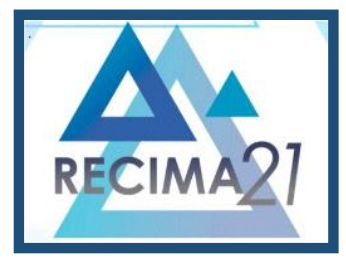

\title{
RECIMA21 - REVISTA CIENTÍFICA MULTIDISCIPLINAR ISSN 2675-6218
}

\author{
INCIDÊNCIA E REPERCUSSÃO DO CÂNCER DE COLO UTERINO EM MULHERES NEGRAS \\ NO MUNICÍPIO DE QUEIMADOS - RJ \\ NO MUNICIPIO DE QUEIMADOS - RJ
Leandro Mendes Martins, Adriana de Aquino Oliveira, Maria Clara Veiga da Rocha, Amanda de Almeida Floriano, \\ Daniel Virginio, Bruna Porath Azevedo Fassarella, Wanderson Alves Ribeiro, Vanessa Vicente Alves Coutinho
}

como o próprio câncer de colo de útero, consequentemente as mulheres negras vivem em situação de alta vulnerabilidade socioeconômica, sendo um fator preponderante para que as mesmas sejam mais afetadas por essa patologia do que mulheres brancas.

No que tange a luta contra o racismo institucional, estados e munícipios brasileiros vem implementado a Política Nacional de Saúde Integral da População Negra (PNSIPN), que é um compromisso firmado pelo Ministério da Saúde no combate às desigualdades no Sistema Único de Saúde (SUS) e na promoção da saúde da população negra de forma integral, considerando que as iniquidades em saúde são resultados de injustos processos socioeconômicos e culturais em destaque, o vigente racismo - que corroboram com a morbimortalidade das populações negras brasileiras (LÓPEZ, 2012).

Para Ribeiro et al., (2017), a Educação em Saúde, que é definida como meio do qual o conhecimento cientificamente produzido no campo da saúde, intermediado pelos profissionais de saúde, onde atinge a vida cotidiana das pessoas, uma vez que a compreensão dos condicionantes do processo saúde-doença oferece subsídios para a adoção de novos hábitos e condutas de saúde. Possuindo amplitude tanto na área da saúde, quanto para educação, resultando em diferentes formas de entendimento e distintas posições sobre o homem e a sociedade.

Cabe mencionar que a equidade, um dos princípios do SUS, que se evidencia no atendimento aos indivíduos de acordo com suas necessidades, oferecendo mais a quem mais precisa e menos a quem requer menos cuidados. Norteando o reconhecimento das diferenças nas condições de vida e saúde e nas necessidades das pessoas, considerando que o direito à saúde passa pelas diferenciações sociais e deve atender a diversidade (BARROS; SOUSA, 2016).

De acordo com a problemática apresentada, o estudo tem objetivo descrever a experiência frente ao índice de câncer do colo uterino em mulheres negras de 25 a 64 anos.

\section{METODOLOGIA}

Tratou-se de um estudo retrospectivo, por revisão bibliográfica, com abordagem quantitativa, a partir de dados coletados no Painel de Percentual das mulheres negras, do município de Queimados - RJ, pertencente a Região Metropolitana I, que buscaram medidas preventivas ao CCU. Os dados também foram coletados no DATASUS, Plano Municipal de Saúde de Queimados - PMS e ainda, Portal da Transparência com dados do município em questão.

Segundo Lakatos (1991) os estudos exploratórios são investigações de pesquisa empírica cujo objetivo é a formulação de questões ou de um problema, no qual se empregam geralmente procedimentos sistemáticos para a obtenção de observações empíricas ou para as análises de dados, em que são obtidas frequentemente descrições tanto quantitativas quanto qualitativas do objeto de estudo. 


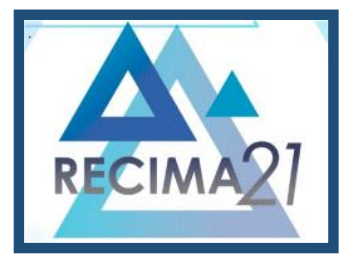

\title{
RECIMA21 - REVISTA CIENTÍFICA MULTIDISCIPLINAR ISSN 2675-6218
}

\author{
INCIDÊNCIA E REPERCUSSÃO DO CÂNCER DE COLO UTERINO EM MULHERES NEGRAS \\ NO MUNICÍPIO DE QUEIMADOS - RJ \\ Leandro Mendes Martins, Adriana de Aquino Oliveira, Maria Clara Veiga da Rocha, Amanda de Almeida Floriano, \\ Daniel Virginio, Bruna Porath Azevedo Fassarella, Wanderson Alves Ribeiro, Vanessa Vicente Alves Coutinho
}

Para Minayo (2014), uma pesquisa exploratória deve seguir os seguintes passos: escolha do tópico de investigação; delimitação do problema, definição do objeto e objetivo, construção do marco teórico conceitual, dos instrumentos de coleta de dados e da exploração do campo.

Para Yin (2001), um relato de experiência pertence ao domínio social, fazendo parte das experiências humanas, devendo conter tanto impressões observadas quanto conjecturadas. Este tipo de estudo é importante para a descrição de uma vivência particular que suscitou reflexões novas sobre um fenômeno específico.

Em relação ao método qualitativo Gil (2008) corrobora que, pode se discorrer que é o processo aplicado ao estudo da biografia, das representações e classificações que os seres humanos fazem a respeito de como vivem, edificam seus componentes e a si mesmos, sentem e pensam.

A abordagem qualitativa na saúde faz emergir questões semelhantes às do âmbito das ciências sociais, pois a saúde não se caracteriza como um campo separado das outras instâncias da realidade social, porém a especificidade da saúde dentro de uma abordagem qualitativa ocorre pelas inflexões socioeconômicas, políticas e ideológicas relacionadas ao saber teórico e prático sobre saúde e doença (MINAYO, 2004).

Para melhor embasamento do estudo foram utilizadas informações do PLANO MUNICIPAL DE SAÚDE DE QUEIMADOS - PMS 2018/2021, Relatórios internos de gestão, ambos disponíveis no portal da transparência do município em questão e ainda, com o recorte e artigos coletados em base de dados virtuais. Para tal utilizou-se a Biblioteca Virtual de Saúde (BVS), na seguinte base de informação: Literatura Latino-Americana e do Caribe em Ciências da Saúde (LILACS), Medical Literature Analysis and Retrieval System Online (MEDLINE) e Scientific Electronic Library Online (SCIELO) dos últimos 10 anos, usando os seguintes descritores: Saúde da Mulher Negra; Racismo; Racismo Institucional; Políticas Públicas e Educação em Saúde, totalizando 6 artigos.

\section{RESULTADOS E DISCUSSÃO}

O Município de Queimados está localizado na Baixada Fluminense, na Região Metropolitana I do Rio de Janeiro, distante cerca de $50 \mathrm{~km}$ da área central da capital. Tem como limites os municípios de Japeri (noroeste), Seropédica (sudoeste) e Nova Iguaçu (norte/nordeste, sul/sudeste). A região se insere como área de expansão da capital, apresentando uma proliferação de loteamentos de baixo custo e carência de infraestrutura, em sua grande maioria, sendo o município caracterizado como cidade-dormitório, cujos habitantes utilizam, em sua grande maioria, a linha férrea e a rodovia Presidente Dutra como principais vias de acesso ao Município do Rio de Janeiro, seu local de trabalho (PMQ/SMS 2018).

Para Freitag (2002, p.4) conforme citado por Ojima et al (2008, p.3) o morador desse tipo de cidade não se reconhece como cidadão da mesma, pois na medida em que ali não se encontra 


\section{RECIMA21 - REVISTA CIENTÍFICA MULTIDISCIPLINAR ISSN 2675-6218}

INCIDÊNCIA E REPERCUSSÃO DO CÂNCER DE COLO UTERINO EM MULHERES NEGRAS NO MUNICÍPIO DE QUEIMADOS - RJ Leandro Mendes Martins, Adriana de Aquino Oliveira, Maria Clara Veiga da Rocha, Amanda de Almeida Floriano,
Daniel Virginio, Bruna Porath Azevedo Fassarella, Wanderson Alves Ribeiro, Vanessa Vicente Alves Coutinho

seu local de trabalho e só serve como dormitório e residência, ele não teria compromisso efetivo com a cidade. A partir desta reflexão podemos considerar também que o trabalhador não exerceria parte de seus direitos e deveres no município para onde se dirige, já que se ocupa estritamente aos afazeres do trabalho. Além disso, a cidade-dormitório teria independência administrativa e econômica relativamente limitada sendo, portanto, altamente dependente da sede regional a qual se vincularia com grande intensidade. Ainda nesse contexto merece destaque as precárias condições de vida para seus moradores que vivenciam altos índices de violência, insalubridade, epidemias, problemas de trânsito e transportes, agressão ao meio ambiente, entre outros.

Dadas todas essas características, a promoção do cuidado a saúde desta parcela da população torna-se um desafio, tendo em vista que na Baixada Fluminense, não se tem como hábito, na garantia de melhoria de acesso, a possibilidade de se trabalhar com o horário estendido (até as 21:00 h) e/ou a abertura das Unidades de Saúde aos finais de semana, salvo os casos de convocação para campanha de vacinação. Fator esse que está diretamente ligado a questão de ajustes em diretrizes municipais, recursos financeiros, aporte de segurança, dentre outros fatores.

O perfil demográfico do município desde a emancipação, em 25 de novembro de 1990, vários fatores econômicos e sociais contribuíram para o crescimento populacional de 13,09\% referentes aos censos de 2000 e 2010 respectivamente. Segundo dados do IBGE, a densidade demográfica é de 1.822,6 habitantes por $\mathrm{km}^{2}$, contra 2.221,8 habitantes por $\mathrm{km}^{2}$ de sua região. A taxa de urbanização corresponde a 100\% da população (PMQ/SMS 2018).

De acordo com o IBGE (2010), a população de Queimados era de 137.962 habitantes e a população em 2017 está estimada em 145.386 habitantes. Ainda segundo o mesmo estudo, a população está distribuída entre as raças branca, preta, parda e indígena. As mulheres apresentam um percentual de 51,7\%; homens, $48,2 \%$ e a população acima de 60 anos representa $9,4 \%$ do total.

Segundo o IBGE o Índice de Desenvolvimento Humano (IDH) de Queimados calculados em 2010, de acordo com o censo, revelou um IDH de 0,680, ocupando o $73^{\circ}$ lugar no estado do Rio de Janeiro, $11^{\circ}$ da Região Metropolitana e $10^{\circ}$ da Baixada Fluminense. O IDHM do município o situa na faixa de Desenvolvimento Humano Médio (IDHM entre 0,600 e 0,699).

A rede municipal de saúde é composta por 40 unidades, cadastradas no sistema sendo 21 públicas municipal, 01 Unidade de Pronto Atendimento Estadual e 06 unidades privadas contratadas de forma complementar, prestando serviço ao SUS.

Das 40 Unidades citadas, 03 são Unidade Básicas de Saúde Tradicional, 05 Equipes de Saúde da Família (ESF) e 08 Clínicas da Família sendo que nestas clinicas 01 atende como UBS tradicional e 01 como modelo misto de assistência, ambas de atendimento com demanda espontânea e as outras 06 Clinicas atendem com Equipes de Saúde da Família (ESF), totalizando $40 \%$ dos serviços de atenção primária a Saúde (PMQ/SMS 2018). 


\section{RECIMA21 - REVISTA CIENTÍFICA MULTIDISCIPLINAR ISSN 2675-6218}

INCIDÊNCIA E REPERCUSSÃO DO CÂNCER DE COLO UTERINO EM MULHERES NEGRAS NO MUNICÍPIO DE QUEIMADOS - RJ Leandro Mendes Martins, Adriana de Aquino Oliveira, Maria Clara Veiga da Rocha, Amanda de Almeida Floriano, Daniel Virginio, Bruna Porath Azevedo Fassarella, Wanderson Alves Ribeiro, Vanessa Vicente Alves Coutinho

Corroborando com o tema, a coordenação do cuidado pela Atenção Primária à Saúde (APS) tem sido apontada internacionalmente como atributo fundamental na organização de sistemas de saúde, para prover melhorias na prestação do cuidado em termos de acesso, qualidade e continuidade da atenção, seja integrando serviços e ações entre diferentes níveis, seja no interior de um mesmo nível do sistema de saúde (ALMEIDA et al, 2012).

A rede secundária possui 04 Unidades cadastradas com especialidades sendo que 01 Unidade é de caráter especializado e regional, ainda no que compete a rede secundária o município dispõe de 04 unidades contratadas de forma complementar ao SUS complementa totalizando $20 \%$ dos serviços disponíveis. A rede terciária é composta por 01 UPA Estadual e 01 unidade de assistência hospitalar infantil, totalizando $5 \%$ dos serviços. Os outros $35 \%$ dos serviços cadastrados incluem Vigilância, setor de Tuberculose, Saúde Mental, Centro de Especialidades de Odontologia e os serviços burocráticos da Secretaria de Saúde (PMQ/SMS 2018).

\section{Gráfico 1 - Divisão por Gênero}

Em relação a divisão por gênero em sua adscrição de clientela, o percentual de mulheres corresponde a $51,57 \%$ e $48,43 \%$ de homens respectivamente, como mostra o gráfico a seguir:

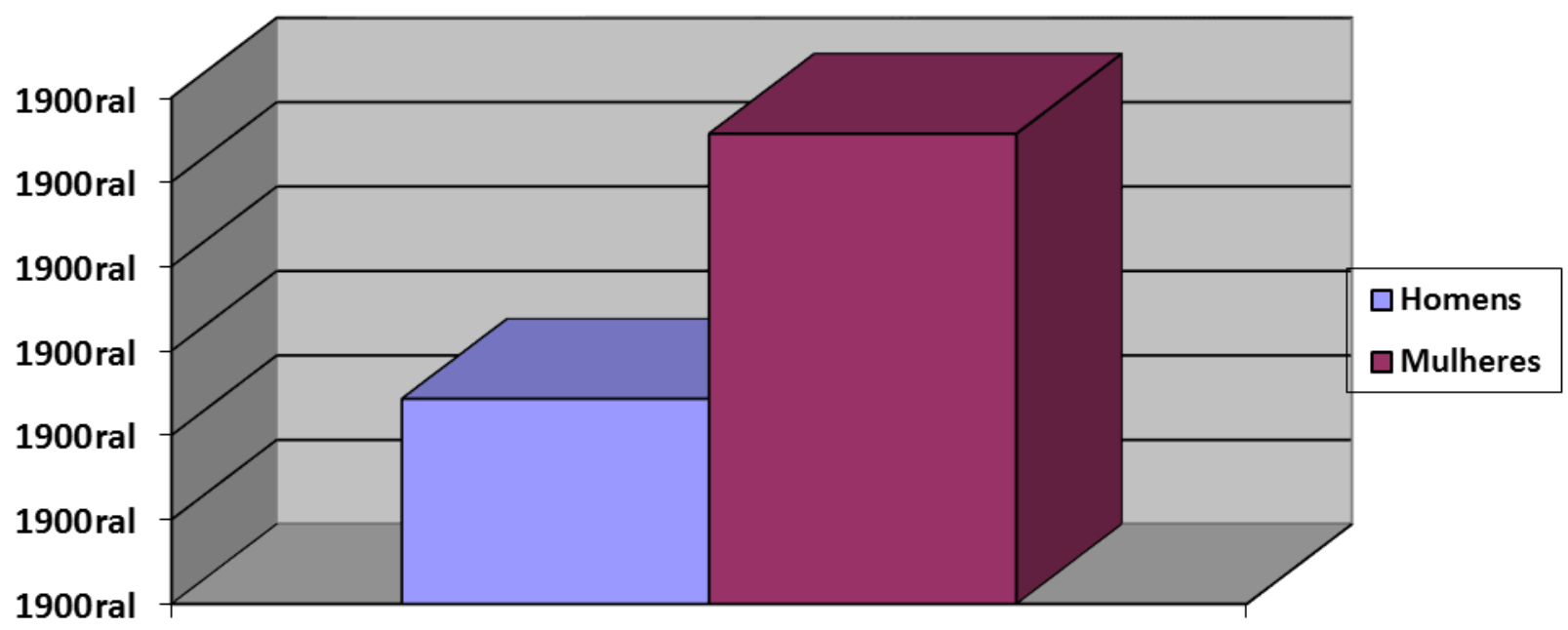




\section{RECIMA21 - REVISTA CIENTÍFICA MULTIDISCIPLINAR ISSN 2675-6218}

INCIDÊNCIA E REPERCUSSÃO DO CÂNCER DE COLO UTERINO EM MULHERES NEGRAS NO MUNICÍPIO DE QUEIMADOS - RJ Leandro Mendes Martins, Adriana de Aquino Oliveira, Maria Clara Veiga da Rocha, Amanda de Almeida Floriano, Daniel Virginio, Bruna Porath Azevedo Fassarella, Wanderson Alves Ribeiro, Vanessa Vicente Alves Coutinho

\section{Gráfico 2 - Divisão por raça}

Quando analisamos a divisão por raça, o percentual de mulheres brancas é 45,94\% e $54,06 \%$ de mulheres negras, conforme gráfico a seguir:

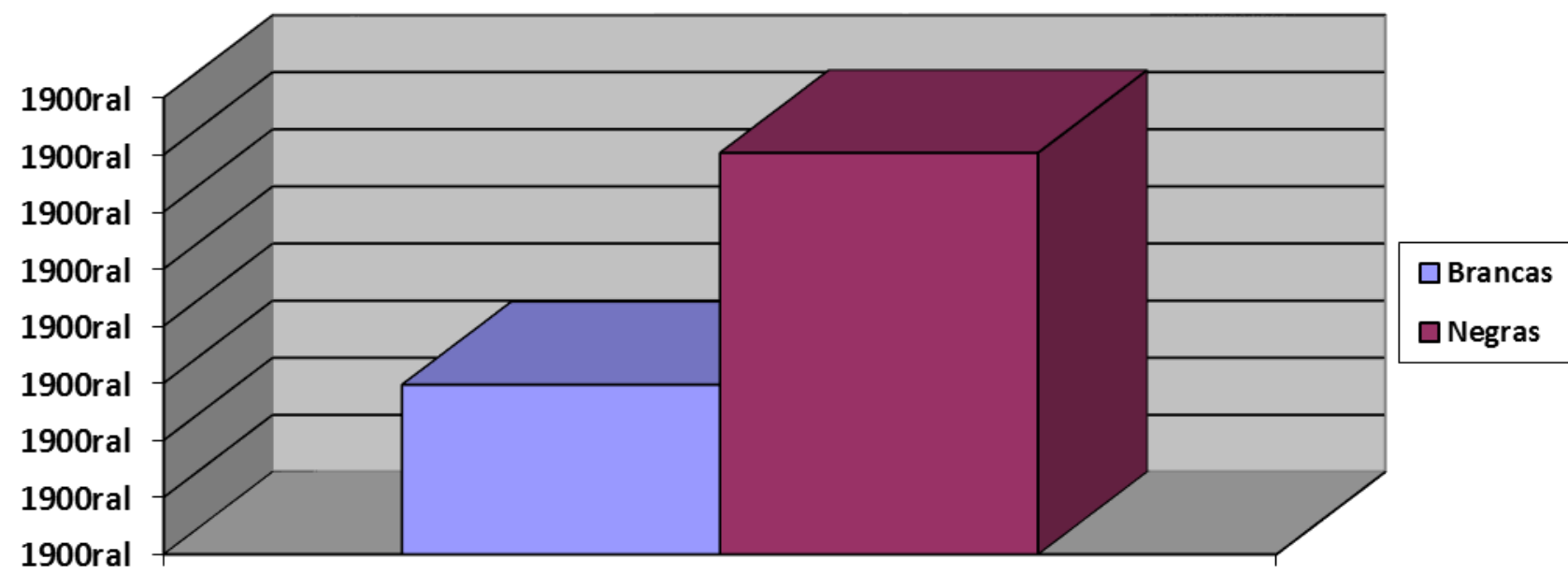

\section{Gráfico 3 - Faixa Etária por raça}

No que tange a mulheres dentro da faixa etária preconizada para realização do Papanicolau a divisão por raça deu-se da seguinte forma: $43,15 \%$ são de Mulheres Brancas e $56,85 \%$ são de Mulheres Negras, de acordo com gráfico a seguir:

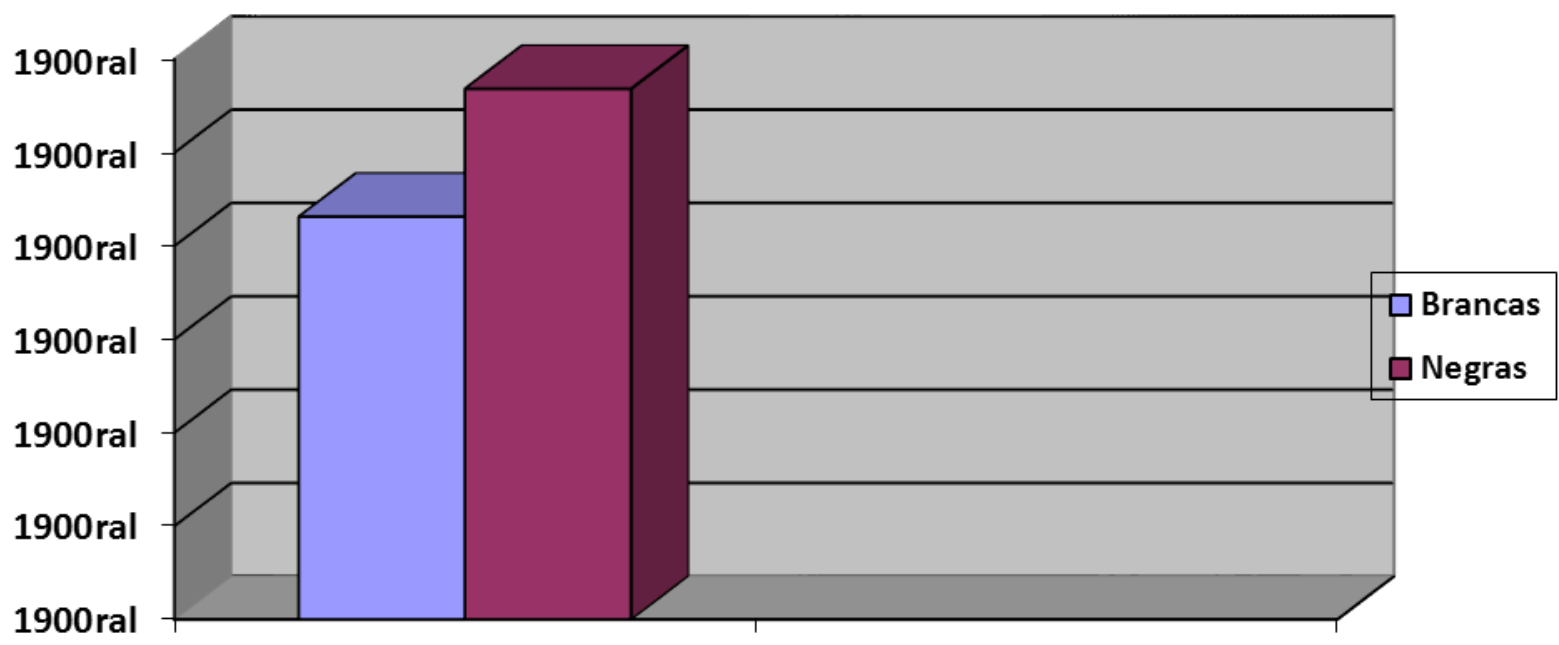

Proporcionalmente, o número de mulheres negras na área da Unidade de Saúde, dentro da faixa etária preconizada para realização do Papanicolau, é superior do que o de mulheres 


\section{RECIMA21 - REVISTA CIENTÍFICA MULTIDISCIPLINAR ISSN 2675-6218}

INCIDÊNCIA E REPERCUSSÃO DO CÂNCER DE COLO UTERINO EM MULHERES NEGRAS NO MUNICÍPIO DE QUEIMADOS - RJ Leandro Mendes Martins, Adriana de Aquino Oliveira, Maria Clara Veiga da Rocha, Amanda de Almeida Floriano, Daniel Virginio, Bruna Porath Azevedo Fassarella, Wanderson Alves Ribeiro, Vanessa Vicente Alves Coutinho

brancas, porém esse maior número não se traduz em maior procura pelos serviços de saúde, o que se torna um desafio na garantia a equidade no acesso ao atendimento.

Para Barros et al (2016) acesso a serviços de saúde e equidade estão intimamente relacionados a capacidade de os sistemas de saúde organizarem-se de modo a responder adequadamente às necessidades dos cidadãos, diferenciando acessibilidade de acesso, onde a primeira refere-se às características da oferta; já o segundo relaciona-se à forma pela qual as pessoas percebem a acessibilidade e ainda que a forma como estes indivíduos percebem a disponibilidade de serviços afeta a decisão de procurá-los, sendo que essa percepção é influenciada pela experiência passada com os serviços de saúde.

\section{Gráfico 4 - Nível de escolaridade das mulheres brancas dentro da faixa etária preconizada para a realização do Papanicolau por raça.}

Cabe mencionar, que ao analisarmos as fichas de cadastro individual do ESUS AB no quesito nível de escolaridade das mulheres na faixa etária preconizada encontramos os seguintes dados: Mulheres Brancas Analfabetas 31,55\%, Mulheres Brancas com Ensino Fundamental Incompleto 41,13\%, Mulheres Brancas com Ensino Médio Completo 22,84\% e 4,48\% de Mulheres Brancas com Ensino Superior Completo e/ou em curso, descritos no gráfico a seguir intitulado: Nível de escolaridade das mulheres brancas dentro da faixa etária preconizada para a realização do Papanicolau por raça.

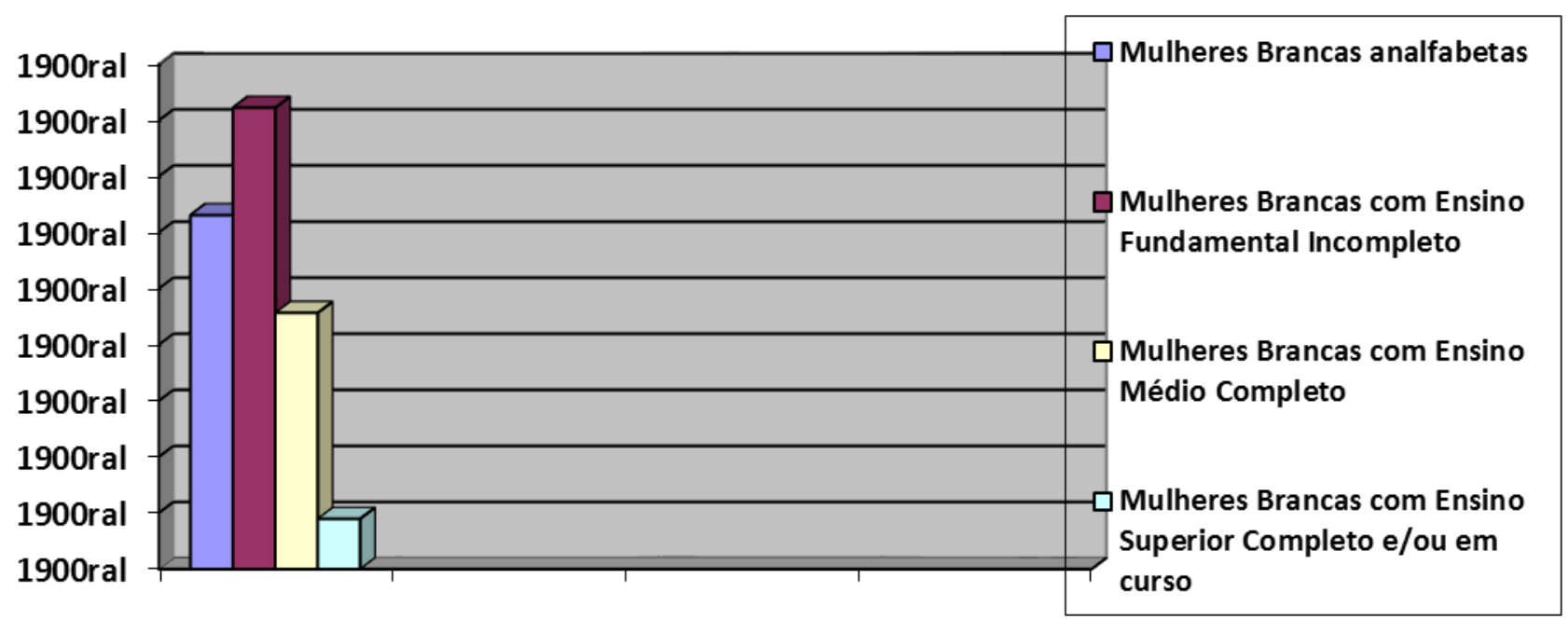




\section{RECIMA21 - REVISTA CIENTÍFICA MULTIDISCIPLINAR ISSN 2675-6218}

INCIDÊNCIA E REPERCUSSÃO DO CÂNCER DE COLO UTERINO EM MULHERES NEGRAS NO MUNICÍPIO DE QUEIMADOS - RJ Leandro Mendes Martins, Adriana de Aquino Oliveira, Maria Clara Veiga da Rocha, Amanda de Almeida Floriano, Daniel Virginio, Bruna Porath Azevedo Fassarella, Wanderson Alves Ribeiro, Vanessa Vicente Alves Coutinho

\section{Gráfico 5 - Nível de escolaridade das mulheres negras dentro da faixa etária preconizada para a realização do Papanicolau por raça.}

Na comparação das variáveis citadas acima, em relação a Mulher Negra, foi constatado que $42,30 \%$ eram analfabetas, 45,79 possuíam o Ensino Fundamental Incompleto, 8,9\% concluíram o Ensino Médio e apenas 3,1\%, conforme o gráfico 5 a seguir intitulado: Nível de escolaridade das Mulheres Negras dentro da faixa etária preconizada para a realização do Papanicolau por raça.

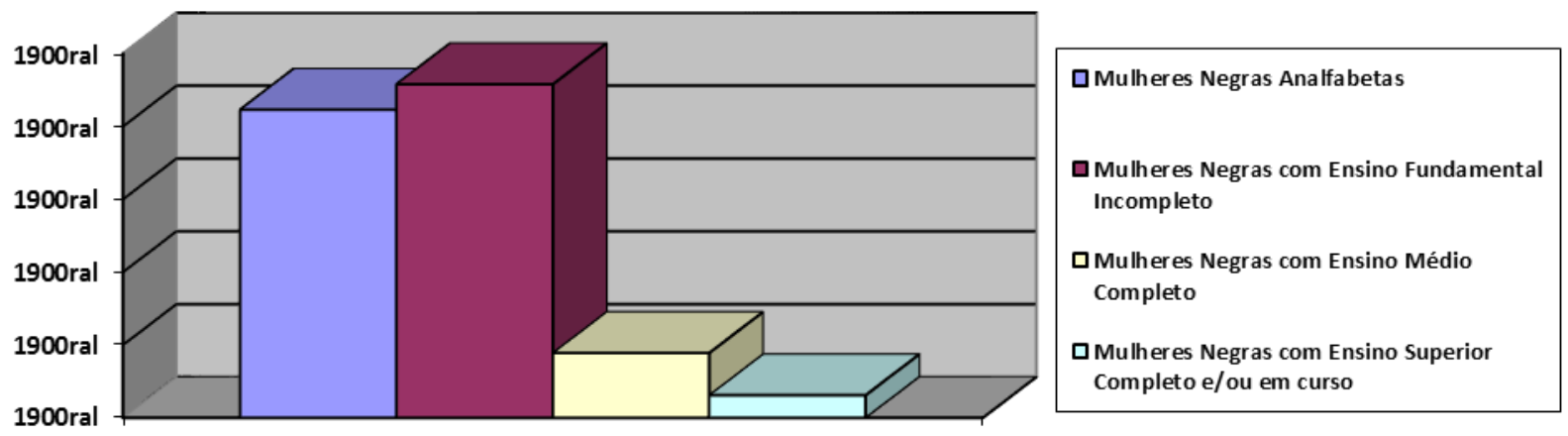

Dadas essas informações, ao analisarmos o percentual de atendimento de mulheres negras no serviço de ginecologia, levamos em consideração os entraves estruturais da organização do serviço municipal e os oriundos as particularidades deste público, tais como: falta de tempo para ir as consultas em virtude de atividades laborais, pudor, déficit de conhecimento da importância e periodicidade para realização de exames ginecológicos, percebemos nitidamente uma procura menor por atendimento da população negra. 


\section{RECIMA21 - REVISTA CIENTÍFICA MULTIDISCIPLINAR ISSN 2675-6218}

INCIDÊNCIA E REPERCUSSÃO DO CÂNCER DE COLO UTERINO EM MULHERES NEGRAS NO MUNICÍPIO DE QUEIMADOS - RJ Leandro Mendes Martins, Adriana de Aquino Oliveira, Maria Clara Veiga da Rocha, Amanda de Almeida Floriano, Daniel Virginio, Bruna Porath Azevedo Fassarella, Wanderson Alves Ribeiro, Vanessa Vicente Alves Coutinho

Gráfico 6 - Percentual de mulheres negras e brancas que realizaram o Papanicolau no período de setembro de 2017 a setembro de 2018

Para demonstrar em números, levando em consideração o recorte temporal o mês 09/2017 a 09/2018, informado nos relatórios internos de gestão, demonstraremos os seguintes dados: números de mulheres negras que realizam o Papanicolau em comparação com as mulheres brancas, o percentual de Lesões intra epiteliais II e III deste grupo e da mesma forma a incidência de câncer de colo uterino no público discutido.

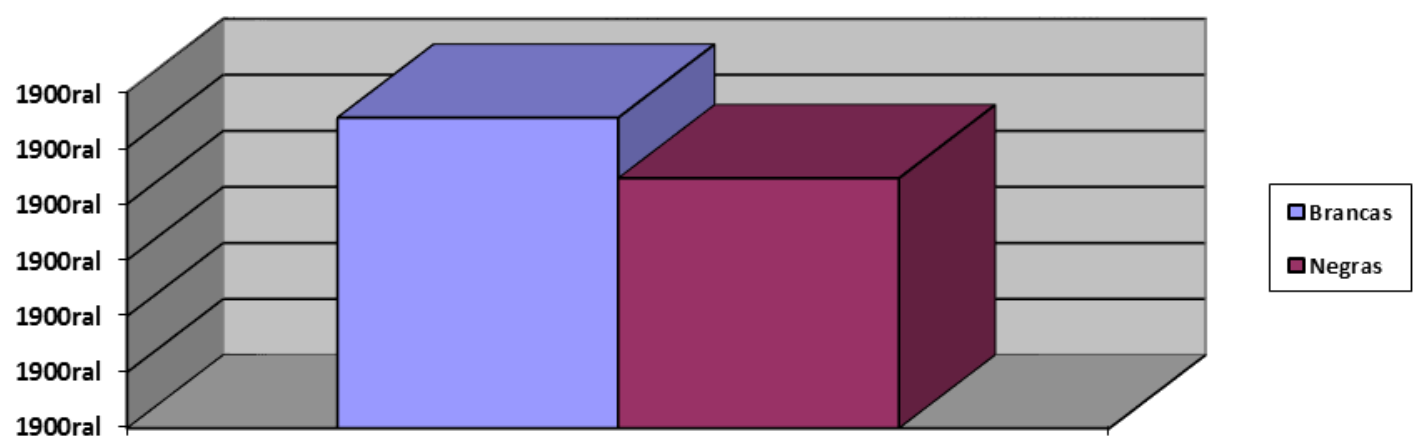

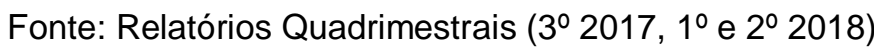




\section{RECIMA21 - REVISTA CIENTÍFICA MULTIDISCIPLINAR ISSN 2675-6218}

INCIDÊNCIA E REPERCUSSÃO DO CÂNCER DE COLO UTERINO EM MULHERES NEGRAS NO MUNICÍPIO DE QUEIMADOS - RJ

Leandro Mendes Martins, Adriana de Aquino Oliveira, Maria Clara Veiga da Rocha, Amanda de Almeida Floriano, Daniel Virginio, Bruna Porath Azevedo Fassarella, Wanderson Alves Ribeiro, Vanessa Vicente Alves Coutinho

\section{Gráfico 7 - Lesões Intra Epiteliais II e III comparadas por raça.}

No período de corte foram realizadas 652 coletas de Papanicolau, onde 55,4\% eram de mulheres brancas e 44,6 de mulheres negras, conforme gráfico 6 . Ao elencarmos os resultados destes exames, no que tange as Lesões Intra-epiteliais II e III somados observamos que dos 130 casos, $58,92 \%$ eram em mulheres negras e $41,08 \%$ eram em mulheres brancas, conforme demonstrada no gráfico 7, intitulado: Lesões Intra Epiteliais II e III comparadas por raça.

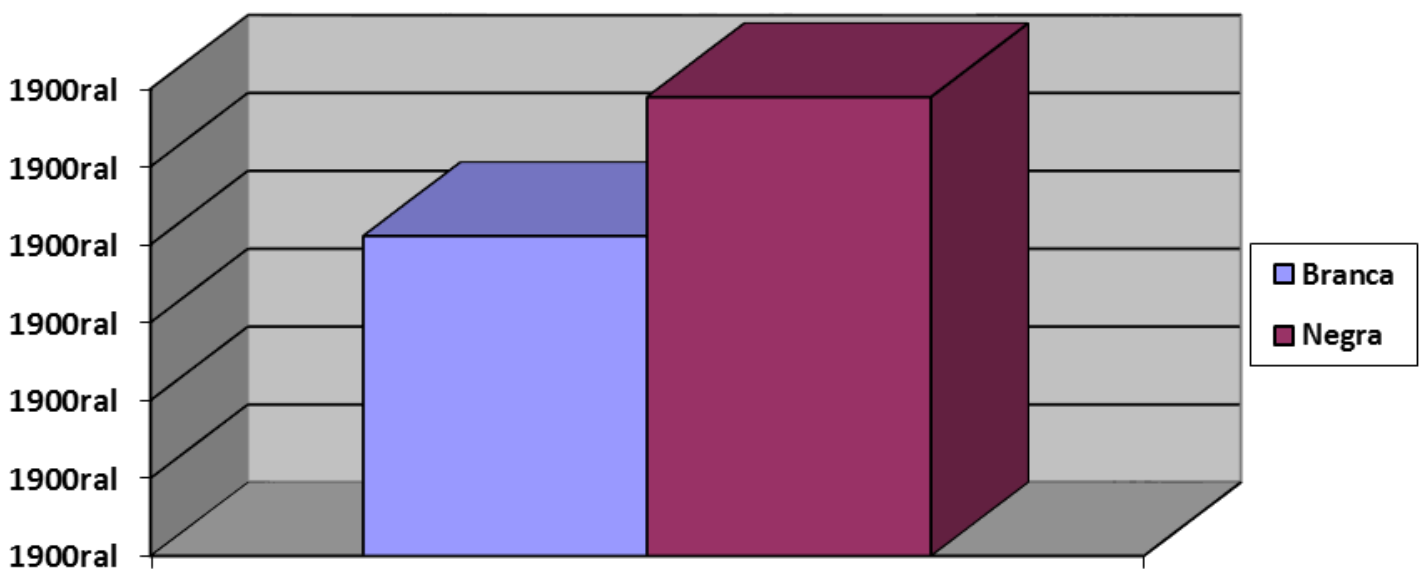

Fonte: Relatórios Quadrimestrais (3ํㅜ 2017, 1ํe 2ํㅜ 2018)

$\mathrm{Na}$ área adscrita no momento temos 02 casos de câncer de colo uterino diagnosticados, que são em mulheres negras e essas estão em tratamento.

\section{Plano de Intervenção: Estratégias e Perspectivas}

Avaliando os entraves citados, parte-se para duas vertentes para a resolução da problemática. No que tange o aspecto organizacional do sistema municipal de saúde faz-se necessário o efetivo aumento de cobertura do território por equipes de Saúde da Família.

Nesse sentindo Arantes et al (2016), reafirmam que a implantação da ESF contribui para a ampliação das possibilidades de oferta de serviços nas áreas periféricas e rurais, onde a mesma traz um efeito estruturante perante os vazios assistenciais em pequenos municípios, com resultados melhores quanto à integralidade da atenção e ao contato por ações programáticas. Também há que se destacar os benefícios da ESF para a promoção da saúde, a prevenção de doenças, a busca ativa de casos, a educação em saúde, a assistência domiciliar, o aumento do número de consultas pré-natais, puericultura, de orientações sobre o aleitamento materno 


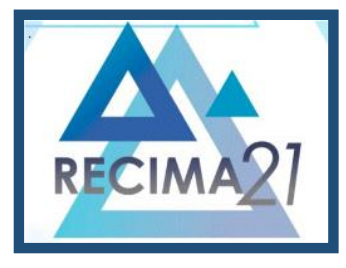

\section{RECIMA21 - REVISTA CIENTÍFICA MULTIDISCIPLINAR ISSN 2675-6218}

exclusivo, da coleta de colpocitologia oncótica; redução de nascidos com baixo peso, da mortalidade infantil e das internações hospitalares.

Com essa expansão a migração de munícipes de outras localidades de vazios assistenciais para áreas cobertas diminuiria, fazendo com que haja a utilização racional das vagas para atendimento aos usuários adscritos do território.

Outro aspecto que merece atenção é a construção do vínculo entre profissionais e usuários, tendo em vista que o vínculo pressupõe a existência de uma fonte regular de atenção, cuja utilização ao longo do tempo requer o estabelecimento de laços interpessoais que reflitam a cooperação mútua entre usuários e profissionais de saúde. Sá et al (2011) diz que a atenção deve produzir-se de relações de escuta, diálogo, respeito, na qual usuário e família possam entender a significância do cuidado e sua corresponsabilidade neste processo. Nessa construção de vínculo sentimentos como pudor, medo entre outros são substituídos por confiança e consequentemente refletem em um maior cuidado à saúde.

Em relação ao déficit de conhecimento por parte das usuárias a educação em saúde é uma ferramenta de grande serventia para a produção do cuidado. A mesma é considerada como um artefato capaz de produzir ação, é um processo de trabalho dirigido para atuar sobre 0 conhecimento das pessoas, para que ocorra desenvolvimento de juízo crítico e capacidade de intervenção sobre suas próprias vidas, ou seja, apropriação da existência como ser humano (BRASIL, 2009).

A prerrogativa de associar a Educação em Saúde nas práticas de Promoção da Saúde pode ser observada como parte integrante das Políticas Públicas na área da Atenção Básica em Saúde, pois o poder público, em suas instâncias federal, estadual e municipal, preconiza a Educação em Saúde e a Promoção da Saúde nos diversos documentos por eles elaborados.

$\mathrm{Na}$ Enfermagem a educação em saúde pode ser definida como um instrumento fundamental para subsidiar uma assistência eficaz e, por estar relacionada diretamente com o autocuidado, com objetivo de proporcionar bem-estar e a qualidade de vida diante de qualquer patologia.

Assim, cabe dizer que a educação em saúde representa uma estratégia fundamental no processo de formação de comportamentos e atitudes que resultem em uma qualidade de saúde, pois, a falta de informação e conhecimento ofertado a população, interfere neste processo e impede à construção de cuidados voltados a saúde.

Resende (2010) afirma que apenas o acesso ao conhecimento, não garante mudança no comportamento cotidiano, este é essencial para desenvolver interesse do indivíduo. Para tanto os fatores determinantes e condicionantes como a alimentação e nutrição, a moradia, o saneamento básico, o meio ambiente, a atividade laboral, a renda, educação, transporte, lazer e o acesso aos bens e serviços necessários devem ser considerados individualmente dentro de qualquer processo de educação em saúde. 


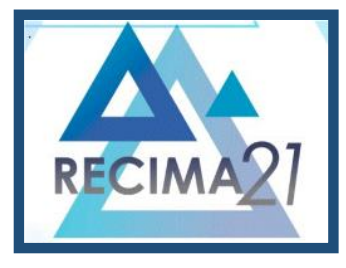

\title{
RECIMA21 - REVISTA CIENTÍFICA MULTIDISCIPLINAR ISSN 2675-6218
}

\author{
INCIDÊNCIA E REPERCUSSÃO DO CÂNCER DE COLO UTERINO EM MULHERES NEGRAS \\ NO MUNICÍPIO DE QUEIMADOS - RJ \\ Leandro Mendes Martins, Adriana de Aquino Oliveira, Maria Clara Veiga da Rocha, Amanda de Almeida Floriano,
Daniel Virginio, Bruna Porath Azevedo Fassarella, Wanderson Alves Ribeiro, Vanessa Vicente Alves Coutinho
}

Como estratégia a prevenção das lesões intra epiteliais e posteriormente CCU cita-se ainda a constante orientação sobre a importância e periodicidade da realização do Papanicolau, a intensificação da vacinação contra o HPV, que protege contra quatro subtipos mais oncogênicos desse vírus $(6,11,16$ e 18) e com esquema completo garante $98 \%$ de eficácia.

De forma geral seria importante a implementação por parte do Município de Queimados, dentro de seus Programas de Atenção Primária a Saúde, a Área Técnica de Atenção Integral a Saúde da População Negra, como forma de fomentar, ações longitudinais em consonância a outras áreas técnicas a esse público alvo.

\section{CONSIDERAÇÕES FINAIS}

Historicamente a população negra sofre discriminação em todos os aspectos sociais, no campo da saúde as dificuldades de acesso aos serviços perpassam pelo viés organizacional dos mesmos, dada aspectos técnicos de gestão, comuns na Baixada Fluminense, que, entretanto, diferem de outras localidades. A interferência política por vezes se sobrepõem a aspectos técnicos, onde a resolutividade de ações será condicionada a expertise do profissional que está executando o cuidado e ainda a implantação de novas unidades para aumento de cobertura obedecem a critérios não claros para os profissionais e população.

Em contraponto a lógica que com o aumento de cobertura territorial com a Estratégia Saúde da Família a ampliação das possibilidades de oferta de serviços nas áreas periféricas e rurais, onde a mesma traz um efeito estruturante perante os vazios assistenciais em pequenos municípios, com resultados melhores quanto à integralidade da atenção e ao contato por ações programáticas.

Ressalta-se que a falta de insumos é uma realidade que dificulta a integralidade da assistência a ser prestada, que acrescida a característica de cidade dormitório, torna-se um desafio para equipes de Saúde da Família. Analisando as dificuldades comumente citadas o pudor, a escassez de tempo para cuidar da saúde e o déficit de conhecimento sobre patologias remetem as equipes a criarem estratégias na garantia do cuidado ao público alvo.

Dentre estas destacam-se o processo de educação em saúde, baseado na capacidade de produção de juízo crítico e capacidade de intervenção em suas próprias vidas que é capaz de produzir mudanças significativas à vida das pessoas, e está intimamente ligada às práticas de Promoção à Saúde, sendo parte integrante das Políticas Públicas na área da Atenção Básica em Saúde.

Além disso estratégias como vacinação contra HPV, implantação da Área Técnica de Atenção Integral a Saúde da População Negra com ações em consonância às demais área temáticas produziriam mudanças circunstanciais na vida dos indivíduos. 


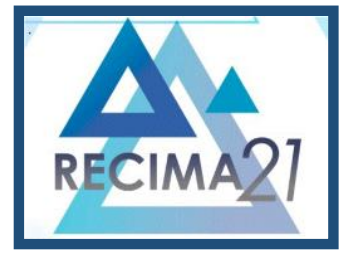

\section{RECIMA21 - REVISTA CIENTÍFICA MULTIDISCIPLINAR ISSN 2675-6218}

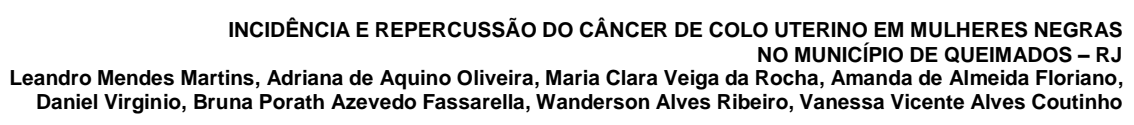

Com este trabalho, ainda se evidencia a necessidade de um estudo de campo, mais abrangente para que a partir dos resultados obtidos possam-se efetuar as mudanças necessárias nas Políticas Públicas de Saúde.

\section{REFERÊNCIAS}

ALMEIDA, P. F.; GIOVANELLA, L.; NUNAN, B. A. Coordenação dos cuidados em saúde pela atenção primária à saúde e suas implicações para a satisfação dos usuários. Saúd em Debate, v. 36, n. 94, p. 375-391, 2012.

ARANTES, L. J.; SHIMIZU, H. E.; MERCHÁN-HAMANN, E. The benefits and challenges of the Family Health Strategy in Brazilian Primary Health care: a literature review. Cienc Saude Colet. v. 21, n. 5, p. 1499-510, 2016.

BARROS, F. P. C.; SOUSA, M. F. Equidade: seus conceitos, significações e implicações para o SUS. Saúde Soc. São Paulo, v. 25, n. 1, p. 9-18, 2016.

BARROS, F. P. C.; LOPES, J. S.; MENDONÇA, A. V. M.; SOUSA, M. F. Acesso e equidade nos serviços de saúde: uma revisão estruturada. Saúde Debate, Rio de Janeiro, v. 40, n. 110, p. 264271, jul./set. 2016.

BATISTA, L. E. et al. Mortalidade da população negra adulta no Brasil. In: BRASIL. Fundação Nacional de Saúde. Saúde da população negra no Brasil: contribuições para a promoção da eqüidade. Brasília, DF: Funasa, 2005. p. 237-314.

BRASIL. MINISTÉRIO DA SAÚDE. DATASUS. Sistema de Informação de Mortalidade (SIM). 2008 Disponível em: http://tabnet.datasus.gov.br/cgi/idb2006/matriz.htm. Acesso em: 30 out. 2018.

CONFERÊNCIA NACIONAL DE SAÚDE ON-LINE. Relatório final. Brasília: Ministério da Saúde; 1996. Disponível em: http://www.datasus.gov.br/cns/temas/educacaosaude/educacaosaude.htm. Acesso em: 30 out. 2018.

CARDOSO, A. M., SANTOS, R. V.; COIMBRA JÚNIOR, C. E. A. Mortalidade infantil segundo raça/cor no Brasil: o que dizem os sistemas nacionais de informação? Cadernos de Saúde Pública, v. 21, n. 5, p. 1602-1608, 2005.

CASADO, L.; VIANNA, L. M.; THULER, L. C. S. Fatores de risco para doenças crônicas não transmissíveis no Brasil: uma revisão sistemática. Rev bras cancerol. v. 55, n. 4, p. 379-88, 2009.

CHAMBÔ FILHO, A.; COHEN, M. C. P. M.; CARDOSO, P. S. Câncer de colo, estádio IB: alternativas de tratamento. Femina, v. 29, n. 9, p. 631-3, 2001.

CHOR, D.; LIMA, A. R. C. Aspectos epidemiológicos das desigualdades raciais no Brasil. Cad Saude Publica, v. 21, n. 5, p. 1586-94, 2005.

COIMBRA, C. E. A.; SANTOS, R. V. Saúde, minorias e desigualdade: algumas teias de interrelações, com ênfase nos povos indígenas no Brasil. Cien Saude Colet, v. 5, n. 1, p. 125-132, 2000.

CORDEIRO, R. C.; FERREIRA, S. L. Discriminação racial e de gênero em discursos de mulheres negras com anemia falciforme. Esc Anna Nery Rev Enferm [online], v. 13, n. 2. abr./jun. 2009. 


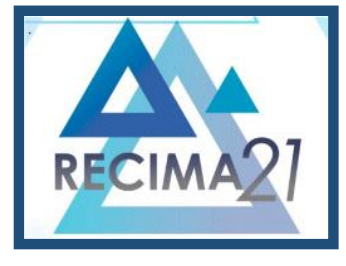

\title{
RECIMA21 - REVISTA CIENTÍFICA MULTIDISCIPLINAR ISSN 2675-6218
}

\author{
INCIDÊNCIA E REPERCUSSÃO DO CÂNCER DE COLO UTERINO EM MULHERES NEGRAS \\ NO MUNICÍPIO DE QUEIMADOS - RJ \\ Leandro Mendes Martins, Adriana de Aquino Oliveira, Maria Clara Veiga da Rocha, Amanda de Almeida Floriano, \\ Daniel Virginio, Bruna Porath Azevedo Fassarella, Wanderson Alves Ribeiro, Vanessa Vicente Alves Coutinho
}

Disponível em: http://www.eean.ufri.br/revista enf/20092/artigo\%2014.pdf. Acesso em: 08 jun. 2018.

GIL, A. C. Métodos e técnicas de pesquisa social. 6. ed. São Paulo: Atlas, 2008.

INSTITUTO BRASILEIRO DE GEOGRAFIA E ESTATÍSTICA (IBGE). Censo Demográfico 2010: resultados do universo. Disponível em http://www.ibge.gov.br/home/estatistica/populacao/censo2010/default resultados universo.sht. Acesso em: 14 ago 2018.

LAKATOS, E. M. Fundamentos de metodologia científica. 3.ed. São Paulo: Atlas, 1991.

LEONE, E. T.; BALTAR, P. A mulher na recuperação recente do mercado de trabalho brasileiro. R. Bras. Est. Pop., São Paulo, v. 25, n. 2, p. 233-249, jul./dez. 2008.

LEOPARDI, M. T. Fundamentos gerais da produção científica. In.: LEOPARDI, M. T (org.). Metodologia da pesquisa em saúde. Santa Maria: Palotti, 2001.

LINARD, A.; SILVA, F. A. D.; SILVA, R. M. Mulheres submetidas a tratamento para câncer de colo uterino - percepção de como enfrentam a realidade. Revista Brasileira de Cancerologia, v. 48, n. 4, p. 493-498, 2020.

LOPES, F. Experiências desiguais ao nascer, viver, adoecer e morrer: tópicos em saúde da população negra no Brasil. In.: BRASIL. Fundação Nacional de Saúde. Saúde da população negra no Brasil: contribuições para a promoção da equidade. Brasília: Funasa; 2005. p. 446.

LÓPEZ, L. C. The concept of institutional racism: applications within the healthcare field. Interface - Comunic. Saude, Educ., v. 16, n. 40, p.121-34, jan./mar. 2012.

LOUREIRO, M. M.; ROZENFELD, S. Epidemiologia de internações por doença falciforme no Brasil. Revista de Saúde Pública, São Paulo, v. 39, n. 6, p. 943-949, dez. 2005.

MENDONÇA, V. G, LORENZATO, F. R. B.; MENDONÇA, J. G. et al. Mortalidade por câncer do colo do útero: características sociodemográficas das mulheres residentes na cidade de Recife, Pernambuco. Rev Bras Ginecol Obstet. v. 30, n. 5, p. 248-55. 2008. DOI:10.1590/S010072032008000500007.

MINAYO, Maria Cecília de Souza. O desafio do conhecimento: pesquisa qualitativa em saúde. São Paulo: Hucitec, 2014.

MINISTÉRIO DA SAÚDE. Secretaria de Atenção à Saúde. Estimativa 2006: incidência de câncer no Brasil. Rio de Janeiro: INCA, 2005.

MINISTÉRIO DA SAÚDE. INSTITUTO NACIONAL DE CÂNCER. Estimativa 2010: incidência de câncer no Brasil. Rio de Janeiro: INCA, 2009. Disponível em: http://www.inca.gov.br/ estimativa/2010/. Acesso em: 14 ago 2018.

MINISTÉRIO DA SAÚDE. INSTITUTO NACIONAL DE CÂNCER. Plano de ação para redução da incidência e mortalidade por câncer do colo do útero: sumário executivo. Rio de Janeiro: INCA, 2010. Disponível em: http://www1.inca.gov.br/inca/Arquivos/Livro DARAO utero.pdf. Acesso em: 14 ago 2018.

MUNANGA, K. Ata da 17ª reunião especial da Assembléia Legislativa de Minas Gerais. Belo Horizonte, 2012. Disponível em: http://www.almg.gov.br/dia/A2002/06/L150602.htm. Acesso em: ago. 2018. 


\title{
RECIMA21 - REVISTA CIENTÍFICA MULTIDISCIPLINAR ISSN 2675-6218
}

\author{
INCIDÊNCIA E REPERCUSSÃO DO CÂNCER DE COLO UTERINO EM MULHERES NEGRAS \\ NO MUNICÍPIO DE QUEIMADOS - RJ \\ Leandro Mendes Martins, Adriana de Aquino Oliveira, Maria Clara Veiga da Rocha, Amanda de Almeida Floriano, \\ Daniel Virginio, Bruna Porath Azevedo Fassarella, Wanderson Alves Ribeiro, Vanessa Vicente Alves Coutinho
}

OJIMA, R.; PEREIRA, R. H. M.; SILVA, R. B. da. Cidades-dormitório e a mobilidade pendular: espaços da desigualdade na redistribuição dos riscos socioambientais? In: [Anais...] ENCONTRO NACIONAL DE ESTUDOS POPULACIONAIS, 16. Caxambu: ABEP, 2008.

PMQ/SMS - PREFEITURA MUNICIPAL DE QUEIMADOS. SECRETARIA MUNICIPAL DE SAÚDE. Plano Municipal de Saúde 2018-2021. Queimados: SMS, 2018.

PMQ/SMS - PREFEITURA MUNICIPAL DE QUEIMADOS. SECRETARIA MUNICIPAL DE SAÚDE. Relatório de Gestão Mensal. QUEIMADOS: SMS/Prefeitura de Municipal de QUEIMADOS, 2018.

PMQ/SMS - PREFEITURA MUNICIPAL DE QUEIMADOS. SECRETARIA MUNICIPAL DE SAÚDE. Relatório de Gestão 3 Quadrimestre. QUEIMADOS: SMS/Prefeitura de Municipal de QUEIMADOS, 2017.

PMQ/SMS - PREFEITURA MUNICIPAL DE QUEIMADOS. SECRETARIA MUNICIPAL DE SAÚDE. Relatório de Gestão 1 Quadrimestre. QUEIMADOS: SMS/Prefeitura de Municipal de QUEIMADOS, 2018.

PMQ/SMS - PREFEITURA MUNICIPAL DE QUEIMADOS. SECRETARIA MUNICIPAL DE SAÚDE. Relatório de Gestão 2o Quadrimestre. QUEIMADOS: SMS/Prefeitura de Municipal de QUEIMADOS, 2018.

PINHO, A. A.; FRANÇA, J. I. Prevenção. do câncer de colo do útero: um modelo teórico para analisar o acesso e a utilização do teste de Papanicolaou. Rev Bras Saude Mater Infant, v. 3, n. 1, p. 95- 112, jan/mar. 2003.

PROGRAMA DAS NAÇÕES UNIDAS PARA O DESENVOLVIMENTO (PNUD). Desenvolvimento Humano e IDH. Disponível em http://www.br.undp.org/content/brazil/pt/home/idh0.html. Acesso em: 10 out. 2018.

IPEA. Atlas da Vulnerabilidade Social. Disponível em http:/ivs.ipea.gov.br/ivs/pt/. Acesso em: 10 out. 2018.

RESENDE, M. G. A. Atuação do enfermeiro da estratégia saúde da família na abordagem da educação em saúde com crianças e adolescentes no município de Conceição das Alagoas - MG. Nescon Biclioteca Virtual. Disponível em: https://www.nescon.medicina.ufmg.br/biblioteca/imagem/2755.pdf. Acesso em: 10 out. 2018.

RIBEIRO, W. A.; MARIANO, E. S.; CIRINO, H. P.; et al. Educação em saúde aos portadores de hipertensão arterial e diabete mellitus na Estratégia Saúde da Família. Revista PróUniverSUS. V. 08, n. 2, p. 110-114, jun./dez. 2017.

ROSE, P.G.; BUNDY, B. N.; WATKINS, E. B. et al. Concurrent cisplatin-based radiotheraphy and chemotherapy for locally advanced cervical cancer. N Engl J Med 1999;340:1144-53. Apud FRIGATO, S. HOGA, LAK. Assistência à mulher com câncer de colo uterino: o papel da enfermagem. Revista Brasileira de Cancerologia, v. 49, n. 4, p. 209-214, 2003.

SÁ, L. D.; GOMES, A. L.; NOGUEIRA, J. A. et al. Intersectorality and bonding in tuberculosis control in family health. Rev Latino-Am Enferm, 19, n. 2, p. 387-95, 2011.

TULLER, L. C. S. Mortalidade por câncer do colo do útero no Brasil. Rev Bras Ginecol Obstet [online]. v. 30, n. 5, 2008. Disponível em: http://www.scielo.br/pdf/rbgo/v30n5/a02v30n5.pdf. Acesso em: 12 out. 2018. 


\section{RECIMA21 - REVISTA CIENTÍFICA MULTIDISCIPLINAR ISSN 2675-6218}

INCIDÊNCIA E REPERCUSSÃO DO CÂNCER DE COLO UTERINO EM MULHERES NEGRAS NO MUNICÍPIO DE QUEIMADOS - RJ Leandro Mendes Martins, Adriana de Aquino Oliveira, Maria Clara Veiga da Rocha, Amanda de Almeida Floriano, Daniel Virginio, Bruna Porath Azevedo Fassarella, Wanderson Alves Ribeiro, Vanessa Vicente Alves Coutinho

WCN. Cervical câncer: Factures that increase your risk of developing cervical câncer. [S.I.]: Gynecologic Câncer Information, 2000. Disponível em: file://A:/cadecolofatorderisco.htm. Acesso em: 17 jul. 2005.

WERNECK, J. Iniquidades raciais em saúde e políticas de enfrentamento: as experiências do Canadá, Estados Unidos, África do Sul e Reino Unido. In.: BRASIL. FUNDAÇÃO NACIONAL DE SAÚDE. Saúde da população negra no Brasil: contribuições para a promoção da equidade. Brasília: Funasa, 2005. p.315-386.

WORLD HEALTH ORGANIZATION. National cancer control programmes: policies and managerial guidelines. 2. ed. Geneva: WHO, 2002.

YIN, R. K. Estudos de caso: planejamento e métodos. 2. ed. Porto Alegre: Bookman, 2001. 